\title{
A mass supercritical and Sobolev critical fractional Schrödinger system
}

\author{
Meiqi Liu ${ }^{1}$ and Quanqing $\mathrm{Li}^{2}$ \\ ${ }^{1}$ Tsinghua University Department of Mathematical Sciences \\ ${ }^{2}$ Honghe University
}

November 13, 2021

\begin{abstract}

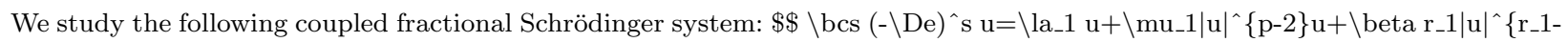
$2\} \mathrm{u}|\mathrm{v}|^{\wedge}\left\{\mathrm{r}_{2} 2\right\} \backslash$ quad $\& \backslash \operatorname{hbox}\{\operatorname{in}\} \backslash ; \backslash \operatorname{mathbb}\{\mathrm{R}\}^{\wedge} \mathrm{N}, \backslash \backslash(-\backslash \mathrm{De})^{\wedge} \mathrm{s} \mathrm{v}=\left.\left|\mathrm{la}_{-} 2 \mathrm{v}+\backslash \operatorname{mu}_{-} 2\right| \mathrm{v}\right|^{\wedge}\{\mathrm{q}-2\} \mathrm{v}+\backslash$ beta $\mathrm{r}_{-} 2|\mathrm{u}|^{\wedge}\left\{\mathrm{r}_{-} 1\right\}|\mathrm{v}|^{\wedge}\left\{\mathrm{r}_{-} 2-2\right\} \mathrm{v} \backslash \mathrm{quad}$ $\& \backslash \operatorname{hbox}\{\operatorname{in}\} \backslash ; \backslash \operatorname{mathbb}\{R\}^{\wedge} N, \backslash \backslash \% \backslash \operatorname{int}\left\{\left\{\backslash \operatorname{mathbb}\{R\}^{\wedge} N\right\} \mathrm{u}^{\wedge} 2=a \backslash\right.$ quad and $\backslash$ quad $\backslash$ int_$\left\{\backslash \operatorname{mathbb}\{\mathrm{R}\}^{\wedge} \mathrm{N}\right\} \mathrm{v}^{\wedge} 2=\mathrm{b}, \backslash$ ecs $\$ \$$ with prescribed mass $\backslash\left[\backslash \operatorname{int}_{-}\left\{\backslash \operatorname{mathbb}\{\mathrm{R}\}^{\wedge} \mathrm{N}\right\} \mathrm{u}^{\wedge} 2=\mathrm{a} \backslash\right.$ quad $\backslash \operatorname{hbox}\{$ and $\} \backslash$ quad $\backslash$ int_ $\left.\left\{\backslash \operatorname{mathbb}\{\mathrm{R}\}^{\wedge} \mathrm{N}\right\} \mathrm{v}^{\wedge} 2=\mathrm{b} . \backslash\right] \mathrm{Here}, \$ \mathrm{a}, \mathrm{b}>0 \$$ are prescribed, $\$ \mathrm{~N}>2 \mathrm{~s}, \mathrm{~s}>\backslash \operatorname{frac}\{1\}\{2\} \$, \$ 2+\backslash \operatorname{frac}\{4 \mathrm{~s}\}\{\mathrm{N}\} 0 \$$ sufficiently large, a mountain pass-type normalized solution exists provided $\$ 2 \backslash$ leq $\mathrm{N} \backslash$ leq $4 \mathrm{~s} \$$ and $\$ 2+\backslash$ frac $\{4 \mathrm{~s}\}\{\mathrm{N}\}$
\end{abstract}

\section{Hosted file}

Manuscript.pdf available at https://authorea.com/users/445923/articles/545301-a-masssupercritical-and-sobolev-critical-fractional-schr\%C3\%B6dinger-system 